\title{
Two new species of Euryhaliotrema Kritsky et Boeger, 2002 (Monogenea: Dactylogyridae) from Lutjanus russellii (Bleeker) and L. argentimaculatus (Forsskål) (Teleostei: Lutjanidae) in the South China Sea
}

\author{
Yuan Sun and Tingbao Yang \\ Correction to: Folia Parasitologica (2015) 62: 040; 10.14411/fp.2015.040; published online 22 July 2015.
}

After the publication of this paper, two members of the editorial board noticed an error in the incorrect assignment of authorities to specific species. The authors and Editors would like to apologise for this mistake.

The correct assignment of taxon authorities are follows:

\begin{tabular}{|c|c|c|}
\hline Position & Incorrect name & Correct name \\
\hline Page 1, Introduction, line 6 & Euryhaliotrema anguiformis Kritsky, 2012 & Euryhaliotrema anguiforme (Zhang in Zhang, Yang et Liu, 2001) Kritsky, 2012 \\
\hline Page 1 , Introduction, line 7 & E. chrysotaeniae Kritsky, 2012 & E. chrysotaeniae (Young, 1968) Kritsky et Boeger, 2002 \\
\hline Page 1, Introduction, line 7 & E. johni Kritsky, 2012 & E. johni (Tripathi, 1959) Kritsky et Boeger, 2002 \\
\hline Page 1, Introduction, line 8 & E. spirotubiforum $\mathrm{Wu}, \mathrm{Zhu}, \mathrm{Xie}$ et $\mathrm{Li}, 2006$ & E. spirotubiforum (Zhang in Zhang, Yang et Liu, 2001) Wu, Zhu, Xie et Li, 2006 \\
\hline Page 1, Introduction, line 14 & E. nanaoense Kritsky, 2012 & E. nanaoense (Li, Yan, Yu, Lan et Huang, 2005) Kritsky, 2012 \\
\hline Page 3 , Remarks, line 4 & E. cribbi Kritsky, 2012 & E. cribbi (Plaisance et Kritsky, 2004) Kritsky, 2012 \\
\hline Page 3, Remarks, line 4 & E. kurodai Kritsky, 2012 & E. kurodai (Ogawa et Egusa, 1978) Kritsky, 2012 \\
\hline Page 3, Remarks, line 5 & E. monoporosum Kritsky, 2012 & E. monoporosum (Pan et Zhang, 2000) Kritsky, 2012 \\
\hline Page 4, Table, column 2 & E. cribbi Kritsky, 2012 & E. cribbi (Plaisance et Kritsky, 2004) Kritsky, 2012 \\
\hline Page 4, Table, column 2 & E. monoporosum Kritsky, 2012 & E. monoporosum (Pan et Zhang, 2000) Kritsky, 2012 \\
\hline Page 4, Table, column 2 & E. nanaoense Kritsky, 2012 & E. nanaoense (Li, Yan, Yu, Lan et Huang, 2005) Kritsky, 2012 \\
\hline Page 4, Table, column 2 & E. spirotubiforum $\mathrm{Wu}, \mathrm{Zhu}, \mathrm{Xie}$ et Li, 2006 & E. spirotubiforum (Zhang in Zhang, Yang et Liu, 2001) Wu, Zh \\
\hline Page 4, Table, column 2 & E. kurodai Kritsky, 2012 & E. kurodai (Ogawa et Egusa, 1978) Kritsky, 2012 \\
\hline Page 4, Table, column 3 & E. annulocirrus Krits & E. annulocirrus (Yamaguti, 1968) Kritsky, 2012 \\
\hline Page 4, Table, c & E. aspistis Kritsky, 2 & E. aspistis (Plaisance et Kritsky \\
\hline Page 4, Table, c & E. berenguelae Kritsk & E. berenguelae (Plaisance et Kritsk \\
\hline Page 4, Table, c & E. grandis Kritsky, 2 & E. grandis (Mizelle et Kritsky, 1969) Kritsky \\
\hline Page 4, Table, c & E. pirulum Kritsky, 2012 & E. pirulum (Plaisance et Kritsky, 2004) Kritsky, 2012 \\
\hline Page 4, Table, c & E. triangulovagina Kritsky, 2012 & E. triangulovagina (Yamaguti, 1968) Kritsky, 2012 \\
\hline Page 4, Table, column 3 & E. longibaculum Kritsky et Boeger, 2002 & E. longibaculum (Zhukov, 1976) Kritsky et Boeger, 2002 \\
\hline Page 4, Table, column 3 & E. mehen Kritsky, 2012 & E. mehen (Soler-Jiménez, Garcia-Gasca et Fajer-Ávila, 2012) Kritsky, 2012 \\
\hline Page 4, Table, column 3 & E. paracanthi Kritsky et Boeger, 2002 & E. paracanthi (Zhukov, 1976) Kritsky et Boeger, 2002 \\
\hline Page 4, Table, column 3 & E. paralonchuri Kritsky et Boeger, 2002 & E. paralonchuri (Luque et Iannacone, 1989) Kritsky et Boeger, 2002 \\
\hline Page 4, Table, column 3 & E. carbunculus Kritsky et Boeger, 2002 & E. carbunculus (Hargis, 1955) Kritsky et Boeger, 2002 \\
\hline Page 4, Table, column 4 & E. microphallus Kritsky, 2012 & E. microphallus (Yamaguti, 1968) Kritsky, 2012 \\
\hline Page 4, Table, column 4 & E. bychowskyi Kritsky et Boeger, 2002 & E. bychowskyi (Obodnikova, 1976) Kritsky et Boeger, 2 \\
\hline Page 4, Table, column 4 & E. anguiformis Kritsky, 2012 & E. anguiforme (Zhang in Zhang, Yang et Liu, 2001) Kritsky, 2012 \\
\hline Page 4, Table, column 4 & E. chrysotaeniae Kritsky et Boeger, 2002 & E. chrysotaeniae (Young, 1968) Kritsky et Boeger, 2002 \\
\hline Page 4, Table, column 4 & E. fastigatum Kritsky et Boeger, 2002 & E. fastigatum (Zhukov, 1976) Kritsky et Boeger, 2002 \\
\hline Page 4, Table, column 4 & E. johni Kritsky et Boeger, 2002 & E. johni (Tripathi, 1959) Kritsky et Boeger, 2002 \\
\hline Page 4, Table, column 4 & E. lutiani Kritsky et Boeger, 2002 & E. lutiani (Yamaguti, 1953) Kritsky et Boeger, 2002 \\
\hline Page 4, Table, column 4 & E. torquecirrus Kritsky et Boeger, 2002 & E. torquecirrus (Zhukov, 1976) Kritsky et Boeger, 2002 \\
\hline Page 4, Table, column 4 & E. tubocirrus Kritsky et Boeger, 2002 & E. tubocirrus (Zhukov, 1976) Kritsky et Boeger, 2002 \\
\hline Page 4, Table, column 4 & E. eukurodai Kritsky, 2012 & E. eukurodai (Zhang, Ding, Lin et Yu, 1994) Kritsky, 2012 \\
\hline Page 5, Remarks, line 17 & E. lutiani Kritsky et Boeger, 2002 & E. lutiani (Yamaguti, 1953) Kritsky et Boeger, 2002 \\
\hline
\end{tabular}

